\title{
OCEBSchool - Sistema de aprendizagem móvel com foco no treinamento para a certificação OCEB
}

\author{
Nemésio Freitas Duarte Filho', Wellington Cristiano ${ }^{2}$ \\ ${ }^{1}$ Instituto Federal de Educação, Ciência e Tecnologia São Paulo (IFSP) \\ Campus São João da Boa Vista - SP - Brasil \\ ${ }^{2}$ Centro Universitário Barão de Mauá \\ Ribeirão Preto - SP - Brasil \\ nemesiodifsp.edu.br, well_rp@hotmail.com
}

\begin{abstract}
Mobile learning applications emerge as an important alternative to enhance teaching and learning, incorporating greater motivation, collaboration and mobility among learners, teachers and tutors. In this context, mobile learning has been used in computer science area, provinding more flexible understanding and dissemination of knowledge in front of students and professionals. Thus, this work presents the development of a mobile learning system with focus on training for OCEB certification. The case study results show that the application helped the approbation of the students, supporting and easing the study and subject of training in question.
\end{abstract}

Resumo. Aplicações de aprendizagem móvel surgem como uma importante alternativa de potencializar o ensino e a aprendizagem, incorporando maior motivação, colaboração e mobilidade entre os aprendizes, professores $e$ tutores. Neste contexto, a aprendizagem móvel vem sendo utilizada na área de informática flexibilizando o entendimento e a disseminação de conhecimento frente a estudantes e profissionais da área. Deste modo, este trabalho apresenta o desenvolvimento de um sistema de aprendizagem móvel com foco no treinamento para a certificação OCEB. Os resultados do estudo de caso demonstram que a aplicação ajudou na provação dos alunos, apoiando e flexibilizando o estudo e treinamento do tema em questão.

\section{Introdução}

Os sistemas e ambientes de aprendizagem têm apresentando uma crescente importância nos últimos anos, tendo um papel fundamental no ensino e treinamento, sendo relevantes não apenas no ambiente acadêmico, mas também no meio industrial (Kinshuk et al. 2003, Duarte et al. 2013).

Esses sistemas juntamente com o advento e desenvolvimento da computação ubíqua vêm proporcionando um novo modelo de aprendizagem, a aprendizagem móvel (m-learning). Este novo modelo proporciona uma maior motivação e interação entre os aprendizes, professores e instrutores, possibilitando aos mesmos contribuir, participar e acessar o sistema de ensino através de dispositivos móveis (Kukulska-Hulme and Traxler 2005). 
Muitos destes sistemas e aplicativos educacionais móveis estão sendo desenvolvidos e elaborados para apoiar o aluno no ensino e no entendimento de temas de diversas áreas (humanas, biológicas e exatas). Este apoio educacional proporciona maior motivação e interatividade para os aprendizes, garantindo maior flexibilidade no ensino (Kinshuk et al. 2003). Neste contexto, verifica-se uma oportunidade para a elaboração de um sistema de aprendizagem móvel para apoiar o treinamento em relação a certificação OCEB (OMG Certified Expert in BPM).

A certificação OCEB - OMG Certified Expert in BPM (Business Process Managment) é uma titulação concedida aos profissionais que demonstram seus conhecimentos frente aos conceitos de gestão de processos de negócio. A certificação OCEB não está vinculada a experiência do profissional, mas sim, na verificação de conhecimentos e diretrizes relacionados com aspectos teóricos de BPM.

Devido à grande variabilidade de informações presentes na certificação OCEB, muitos profissionais de TI (Tecnologia da Informação) apresentam dificuldade de entendimento e, até mesmo, na análise e utilização destes conceitos. Muitos profissionais acabam se direcionando a cursos presenciais, possuindo altos custos financeiros e horários inflexíveis. Por outro lado, aplicar o estudo direcionado a certificação OCEB mediante apostilas e de forma individual, ausente de apoio, pode causar desinteresse e dificuldades de aprendizagem.

Dentro deste contexto, para uma melhor disseminação de conhecimento e uma potencialização na aprendizagem e apoio para os profissionais interessados na certificação OCEB, este artigo apresenta a proposta de desenvolvimento de um sistema de aprendizagem móvel em relação ao treinamento frente aos conceitos e diretrizes necessários para a realização da certificação OCEB. O sistema denominado OCEBSchool foi desenvolvido com a ajuda de especialistas, sendo aplicado na prática com profissionais da área por meio de um estudo de caso. O estudo possibilitou verificar a efetividade da aplicação educacional por meio da porcentagem de aprovações dos usuários que utilizaram o sistema como forma de apoio ao estudo, incorporando também níveis de satisfação por parte dos usuários.

O artigo encontra-se organizado como se segue. A Seção 2 apresenta conceitos sobre aprendizagem móvel e a certificação OCEB. A Seção 3 traz a visão geral do sistema, descrevendo o seu processo de desenvolvimento $\mathrm{e}$ as principais funcionalidades. A Seção 4 apresenta um estudo de caso em relação a utilização prática da aplicação educacional. Por fim, a Seção 5 apresenta a conclusão e trabalhos futuros.

\section{Referencial Teórico}

\subsection{Aprendizagem Móvel (m-learning)}

Com o advento e evolução das tecnologias de informação, juntamente com a computação ubíqua, é possível observar uma nova modalidade de ensino, baseada na computação ubíqua ou computação móvel, denominada aprendizagem móvel ou mobile learning (m-learning) (Zare 2011, Moura 2009).

Por se tratar de um conceito novo e emergente, há diversas tentativas para definir aprendizagem móvel. Rachid and Ishitani (2012) definem aprendizagem móvel como 
qualquer tipo de ensino ou aprendizagem que ocorre quando o aluno não está em algum lugar fixo, ou quando o indivíduo aproveita-se de oportunidades de aprendizagem disponibilizadas pelas tecnologias móveis. Ozdamli and Cavus (2011) abordam aprendizagem móvel como uma atividade que permite aos indivíduos serem mais produtivos quando consomem, criam ou interagem com as informações, mediados por dispositivos digitais móveis e portáteis.

Independentemente da definição adotada, a utilização de sistemas de aprendizagem por meio de dispositivos móveis traz benefícios que vão além de acessibilidade, comodidade e comunicação (Schepman et al. 2012). Por meio dos benefícios proporcionados pela aprendizagem móvel, é possível observar diversos estudos de casos (Unesco 2013) em relação a aplicação e uso da aprendizagem móvel nas diversas áreas de conhecimento, possibilitando maior comodidade, motivação e apoio em relação as práticas educacionais.

\subsection{BPM (Gerenciamento de Processos de Negócios)}

Gerenciamento de processos de negócio (Business Process Managment - BPM) é uma abordagem disciplinada para identificar, desenhar, executar, documentar, monitorar e controlar processos de negócios de forma automatizada ou não, alcançando resultados alinhados com as metas estratégicas de uma organização (Mates 2012).

Segundo Siqueira (2006), o gerenciamento de processos de negócio, é um conceito estratégico para a manipulação de processos. De modo geral, o gerenciamento de processos de negócios utiliza as melhores práticas de gestão, tais como: mapeamento de processos, modelagem, definição de nível de maturidade, documentação, plano de comunicação, automação, monitoramento mediante indicadores de desempenho e ciclo de melhoria. Essas práticas aplicadas ajudam a maximizar os resultados e o desempenho dos processos, permitindo às organizações melhorar a sua rentabilidade, vantagem competitiva, redução de custos, entre outros aspectos.

Devido a competitividade do mercado atual, é prescindível que as organizações tenham o conhecimento claro dos seus processos no seu dia a dia, assim, tornando útil e relevante a utilização dos conhecimentos vinculados ao gerenciamento de processos de negócios tanto por parte da alta direção como por parte de profissionais da área.

\subsubsection{OCEB - OMG Certified Expert in BPM}

A certificação OCEB é uma certificação concedida pela $\mathrm{OMG}^{1}$ (Object Management Group), atualmente ela se encontra em sua segunda versão OCEB2 ${ }^{2}$. É uma certificação onde possui um conjunto de informações relacionados com as práticas do BPM.

A certificação OCEB é na verdade dividida em cinco titulações, distribuídas em três níveis. A certificação básica é a OCEB Fountation. Os dois níveis seguintes são segmentados em conhecimentos técnicos ou de negócio: Business Intermediate e Technical Intermediate, e na sequência, Business Advanced e Technical Advanced.

\footnotetext{
${ }^{1}$ http://www.omg.org/

${ }^{2}$ http://www.omg.org/oceb-2/
} 
V Congresso Brasileiro de Informática na Educação (CBIE 2016)

Anais do XXVII Simpósio Brasileiro de Informática na Educação (SBIE 2016)

Para fazer o exame não há pré-requisitos. Basta estudar a bibliografia relacionada ao respectivo exame, efetuar o pagamento/agendamento e realizar a prova da certificação em um local autorizado para a sua aplicação. Atualmente a certificação OCEB2 se encontra disponível no idioma inglês.

Nesse contexto, devido a grande relevância do mercado por profissionais com conhecimentos em gerenciamento e modelagem de processos negócios, os cursos preparatórios para a certificação OCEB têm sido oferecidos com um alto custo financeiro. A disponibilidade de aplicações gratuitas com um conteúdo educacional apropriado ao estudo e preparação para a certificação OCEB poderia aumentar e flexibilizar o conhecimento em relação a está área específica do mercado de TI.

\section{Visão Geral do OCEBSchool}

A aplicação educacional desenvolvida neste trabalho é um sistema de aprendizagem móvel, cujo objetivo é prover apoio a aprendizagem aos profissionais que buscam fixar/concretizar seus conhecimentos sobre o tema de BPM, apoiando o estudo e realização da certificação OCEB. Os usuários em sua maioria são profissionais da área de TI, sendo também de usufruto aos docentes, possibilitando utilizar o sistema como forma complementar ao conteúdo educacional sobre BPM.

Espera-se que um sistema de aprendizagem móvel específico para a certificação OCEB, possa introduzir aos alunos de forma flexível e motivadora: (1) aspectos didáticos e lúdicos relacionados aos fundamentos e conceitos de gerenciamento de processos de negócio; (2) abordar a notação de modelagem sobre processos de negócios; (3) explicar aspectos relacionados a modelagem e execução de processos de negócios; (4) facilitar a identificação de características presentes em um processo de negócio; e (5) exemplificar o uso do BPM em um contexto real e prático; entre outros aspectos.

O sistema denominado OCEBSchool, desenvolvido neste trabalho, tem por objetivo não somente auxiliar no ensino e aprendizagem desta área, mas também proporcionar novas formas de interação. Para isso, incorpora formas de interatividade com o aluno, facilitando a sua aprendizagem e o entendimento das informações. $\mathrm{O}$ sistema além de apresentar um conteúdo didático e lúdico específico para a área de BPM (com vídeos, imagens, animações, casos reais, entre outros) também tem por objetivo proporcionar maior comodidade e flexibilidade em relação a sua utilização por meio de dispositivos móveis aumentando a motivação por parte dos alunos. Ressalta-se, que todo o conteúdo didático, juntamente com os exercícios e simulados estão disponibilizados em português e em inglês, vendo que o exame da certificação OCEB é realizado sobre o idioma inglês.

\subsection{Processo de Desenvolvimento}

O processo de desenvolvimento do sistema educacional foi definido em quatro etapas:

1.Concepção e Definição do Escopo: a definição do escopo e problemática do trabalho surgiu a partir das necessidades e carências encontradas na comunidade e no meio científico, tendo como foco a certificação OCEB, no qual apresenta carência e precariedade em relação a um sistema de apoio ao ensino e aprendizagem. 
V Congresso Brasileiro de Informática na Educação (CBIE 2016)

Anais do XXVII Simpósio Brasileiro de Informática na Educação (SBIE 2016)

2.Projeto de Interface do Protótipo: a construção de interfaces é uma peça fundamental para um sistema computacional, sendo desejável que ela seja estruturada e adaptada para sistemas móveis. Para esta etapa o escopo do design foi definido por meio das seguintes atividades: (1) definição dos requisitos a serem implementados; (2) definição da infraestrutura de hardware e software para implementação do protótipo; e (3) definição das opções/contrapartidas a serem consideradas como limitações do protótipo.

3.Definição e Desenvolvimento: a construção do protótipo foi realizado sobre o sistema operacional Android, possuindo uma distribuição gratuita. Os requisitos funcionais necessários a serem incluídos no protótipo foram levantados a partir de consultas em trabalhos relacionados à aprendizagem móvel e com a ajuda de especialistas da área de BPM.

4.Avaliação: para evidenciar os resultados finais, foi realizado um estudo de caso com profissionais da área de TI, que utilizaram o OCEBSchool para se preparar frente ao exame de certificação OCEB, verificando a porcentagem de aprovados juntamente com o nível se satisfação por parte dos participantes, destacando também aspectos positivos e negativos do sistema educacional.

\subsection{Principais Características e Funcionalidades}

As principais características e funcionalidades da aplicação educacional são apresentadas a seguir.

Interface Central: após realizar o acesso, é apresentado ao usuário a área central do ambiente (Figura 1) com um menu de tópicos de navegação do curso.

\section{:-1 AppBPM}

Bem-Vindo ao curso de BPM \& BPMN para certificado OCEB Fundation

Para iniciar o curso, basta selecionar a flexa abaixo e selecionar o modulo que irá iniciar

Obrigado e bons estudos

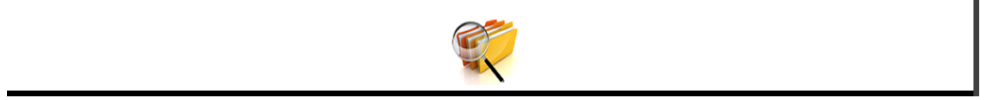

Figura 1: Tela Inicial - OCEBSchool

Modelagem Educacional: no contexto da aprendizagem móvel, é importante evitar interfaces sobrecarregadas e pouco intuitivas principalmente no que se refere ao conteúdo educacional, para isso todo o conteúdo educacional foi projetado seguindo o modelo educacional AIM-CID (Abordagem Integrada de Modelagem - Conceitual, Instrucional e Didática) (Barbosa and Maldonado, 2011). Todo o material foi elaborado por especialistas da área de BPM, sendo segmentado nos seguintes tópicos: "Planejamento do BPM", "Modelagem e otimização de processos", "Execução", "Controle e análise dos dados", "Simulação" e "Exercícios/Simulados". Os tópicos foram divididos com base nos preceitos e diretrizes da certificação OCEB2, possuindo 
V Congresso Brasileiro de Informática na Educação (CBIE 2016)

Anais do XXVII Simpósio Brasileiro de Informática na Educação (SBIE 2016)

uma estrutura progressiva, possibilitando a aprendizagem por níveis básicos, intermediários e avançados.

Ainda em relação à interface principal, encontra-se uma importante região representada por uma barra de botões, que provê funcionalidades específicas ao usuário, sendo elas: (1) Botão Profile, permite a visualização e edição dos dados dos usuários; (2) Botão Search, permite a realização de pesquisas, facilitando a busca ao longo de todo o curso; (3) Botão Refresh, útil pois atualiza as informações na tela quando acionado; e (4) Botão Logout, encerrando a sessão do usuário.

A Figura 2 apresenta algumas telas e aspectos educacionais específicos da aplicação OCEBSchool. Todo o conteúdo foi definido em módulos, facilitando a continuidade do estudo (Figura 2a). A aplicação educacional também possui uma estrutura de "rolagem" dos slides, facilitando a sua utilização e leitura frente a um dispositivo móvel (Figura 2b). Outra característica importante é o "Zoom de Imagem", o conteúdo a ser estudado para a certificação OCEB exige a análise de processos modelados, para isso, é relevante uma funcionalidade específica para a manipulação e aumento da imagem, facilitando o seu entendimento (Figura 2c). Com o objetivo de garantir maior feedback e conhecimento, ao final de cada módulo é proposto uma bateria de testes possibilitando realizar a fixação do conhecimento adquirido, os testes possuem mecanismos de feedback imediato em relação aos erros e acertos. A aplicação educacional também incorpora um módulo específico de simulado, possibilitando uma maior preparação aos candidatos que irão realizar a prova de certificação (Figura 2d).

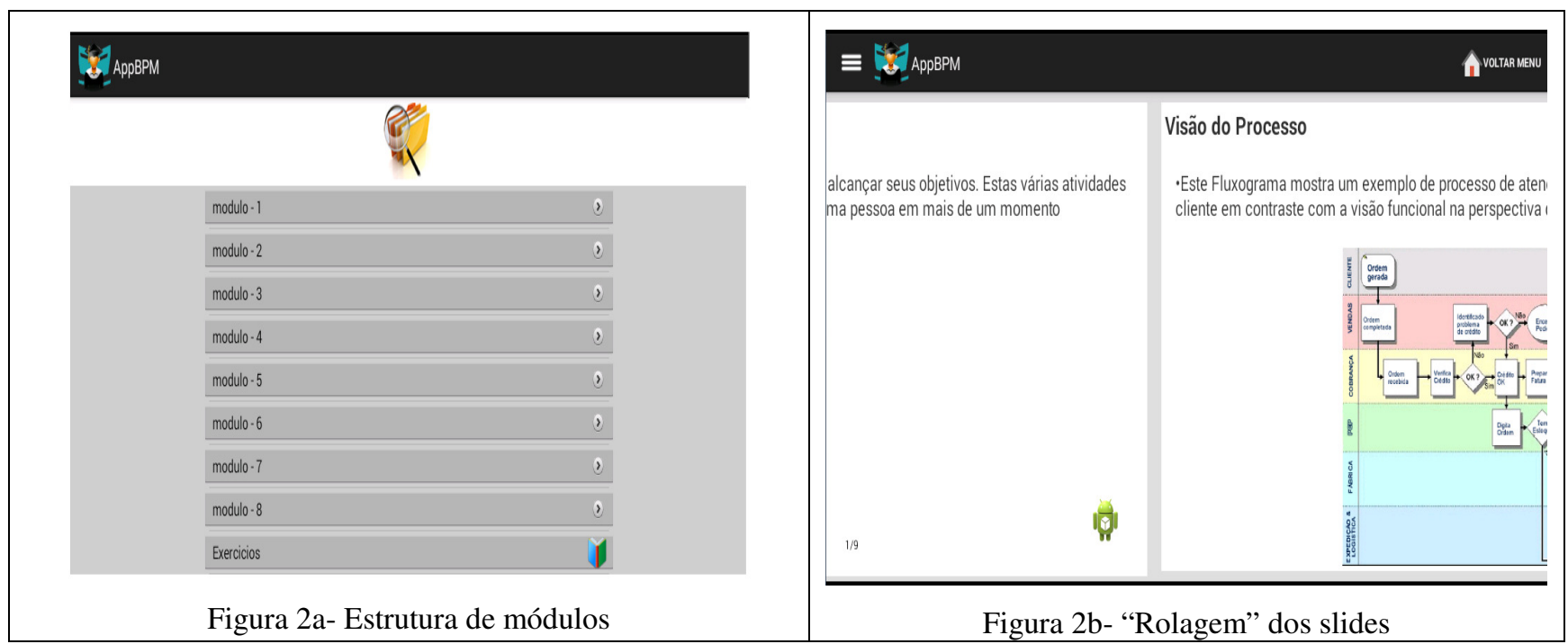


V Congresso Brasileiro de Informática na Educação (CBIE 2016)

Anais do XXVII Simpósio Brasileiro de Informática na Educação (SBIE 2016)

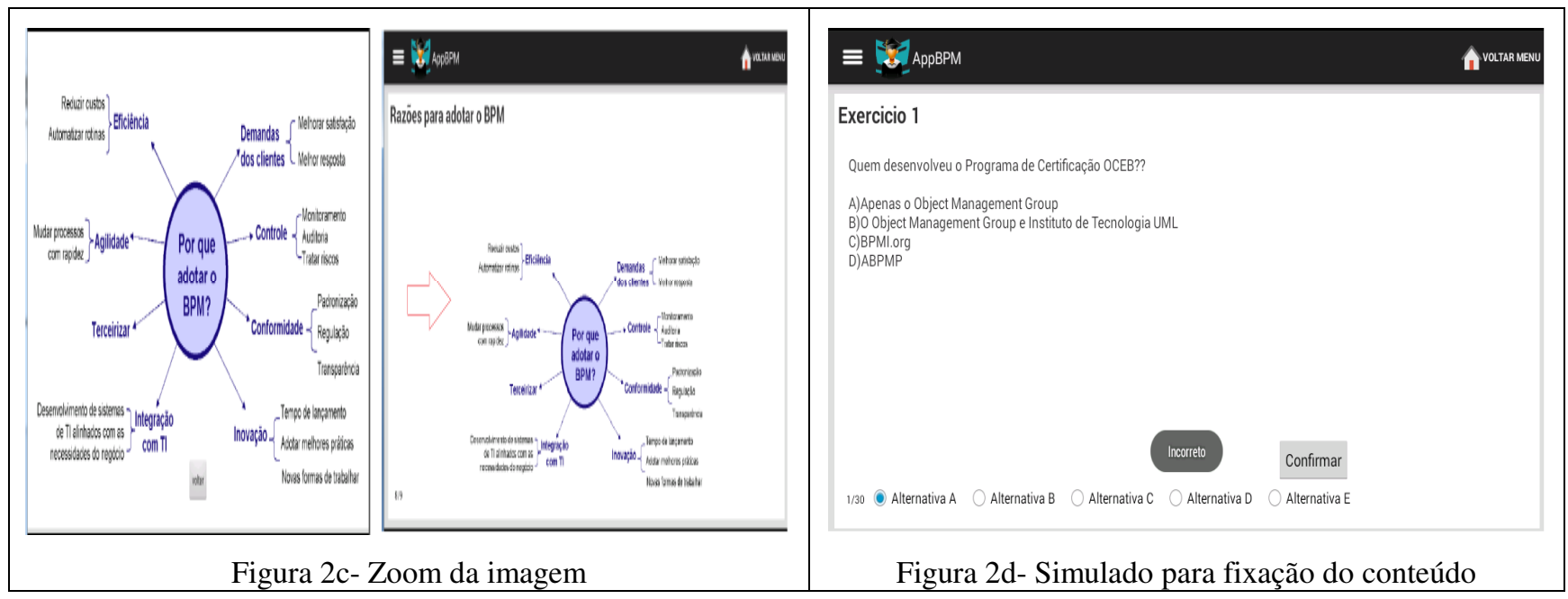

Figura 2: Telas do OCEBSchool

Frente ao contexto da aprendizagem móvel, a aplicação educacional desenvolvida exige um grande cuidado na apresentação do conteúdo para que não haja uma sobrecarga de textos na tela. É relevante evitar que informações desnecessárias sejam disponibilizadas para os usuários. Para isso, algumas páginas possuem um botão com informações complementares (Figura 3). O usuário ao clicar sobre o botão terá acesso, por meio de pop-ups, a informações extras do texto pertinentes ao assunto incorporado na página em questão.

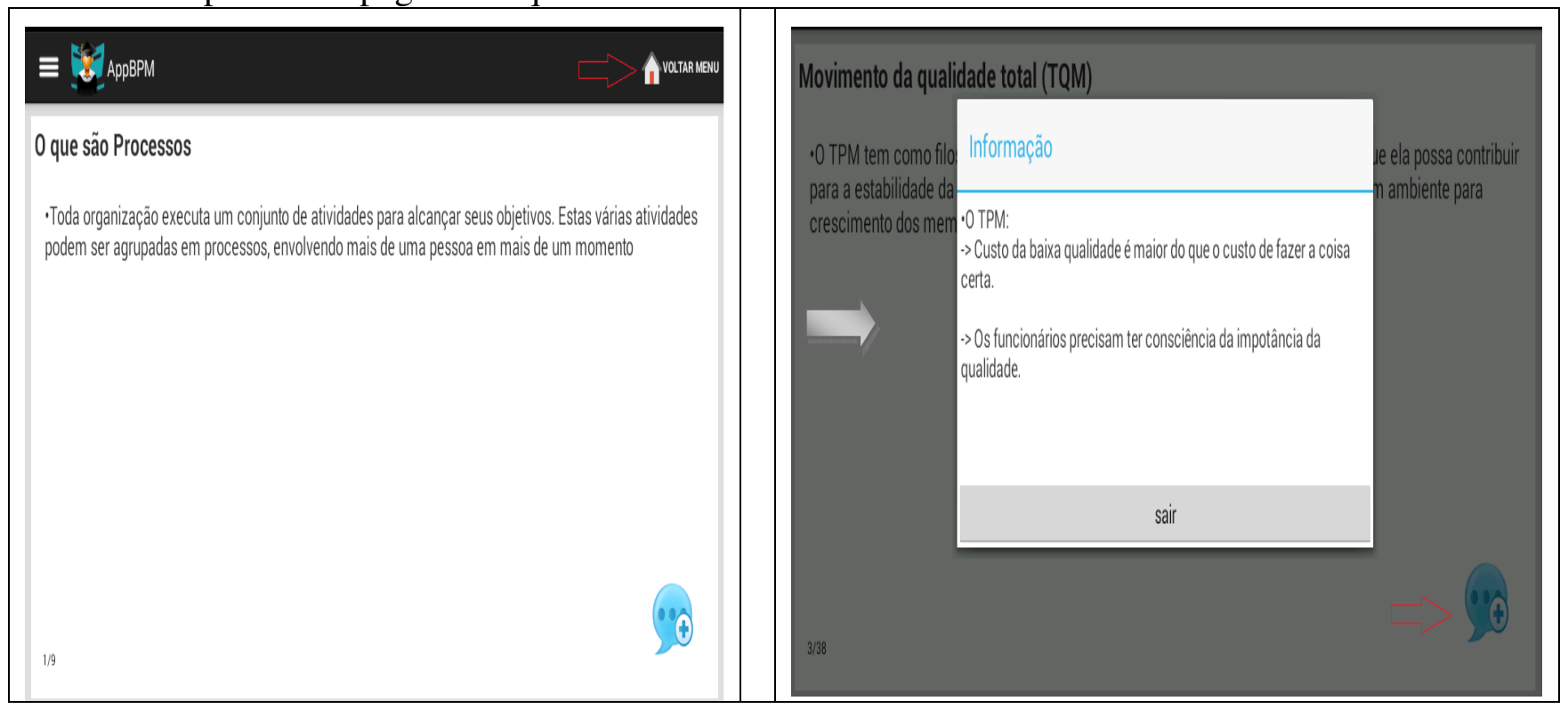

Figura 3: Informações complementares

\section{Estudo de Caso}

A avaliação do aplicativo OCEBSchool foi realizado por meio de um estudo de caso, em um contexto real e prático tendo em vista a participação de profissionais de TI para a preparação da certificação OCEB. O objetivo desta avaliação foi verificar a satisfação dos participantes frente ao uso do aplicativo educacional móvel, verificando ao final de todo o processo o número de candidatos aprovados na certificação OCEB, tendo como apoio a utilização da aplicação OCEBSchool. 
V Congresso Brasileiro de Informática na Educação (CBIE 2016)

Anais do XXVII Simpósio Brasileiro de Informática na Educação (SBIE 2016)

Em um primeiro momento, um grupo de 31 profissionais de TI, participantes de um curso presencial preparatório para a certificação OCEB foram convidados a utilizar o aplicativo OCEBSchool. Os participantes utilizaram a aplicação como forma complementar aos estudos. Todas as instruções de uso, procedimentos, foram passados inicialmente para os participantes, tendo um apoio por parte do tutor do curso presencial.

Ao longo do curso presencial ( 2 meses de duração), os candidatos utilizaram o aplicativo OCEBSchool tanto dentro da sala de aula (direcionado pelo tutor do curso), quanto em suas residências em horários flexíveis realizando atividades completares de estudo. É importante ressaltar, que o conteúdo incorporado no OCEBSchool levou em conta a experiência tanto de profissionais de TI quanto de professores da área em questão, garantindo um conteúdo lúdico de fácil leitura e compreensão, sendo um diferencial para a preparação da certificação.

Ao término do curso presencial, os participantes responderem um formulário eletrônico visando a identificação de níveis de satisfação em relação a utilização do aplicativo educacional, juntamente com a identificação de pontos positivos e negativos. É relevante frisar, que o preenchimento do formulário foi realizado anteriormente a realização da prova de certificação. Esse procedimento foi realizado com a finalidade de evitar que os resultados da certificação (aprovação ou reprovação) pudessem influenciar nas avaliações dos participantes.

Na Figura 4 é possível observar um alto índice de satisfação em relação ao desempenho de utilização do OCEBSchool por parte dos participantes. Os participantes também indicaram pontos positivos em relação a estrutura e o conteúdo didático incorporado no sistema, juntamente com a flexibilidade e comodidade em relação ao uso frente ao ensino e treinamento. Também destacaram alguns pontos negativos e limitações relacionados: (1) ausência de um tutorial auto explicativo (animação), que pudesse ajudar leigos na utilização de aplicações móveis; e (2) ausência de versões nativas do sistema para outros sistemas operacionais móveis, tais como iOS e Windows Phone.

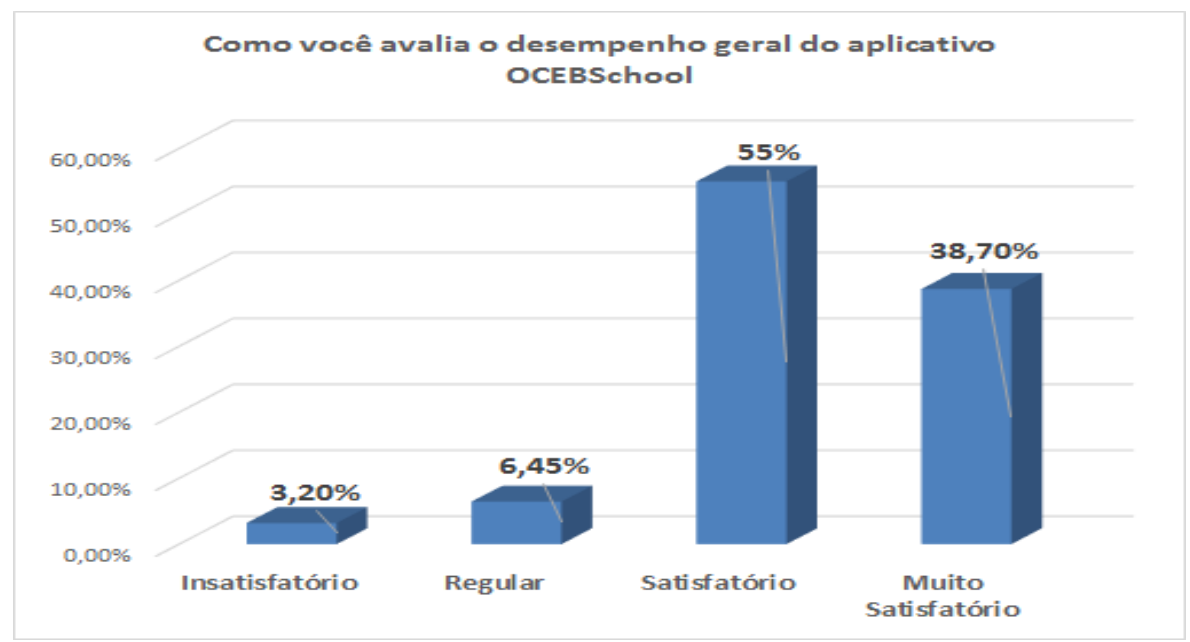

Figura 4: Nível de Satisfação do OCEBSchool 
V Congresso Brasileiro de Informática na Educação (CBIE 2016)

Anais do XXVII Simpósio Brasileiro de Informática na Educação (SBIE 2016)

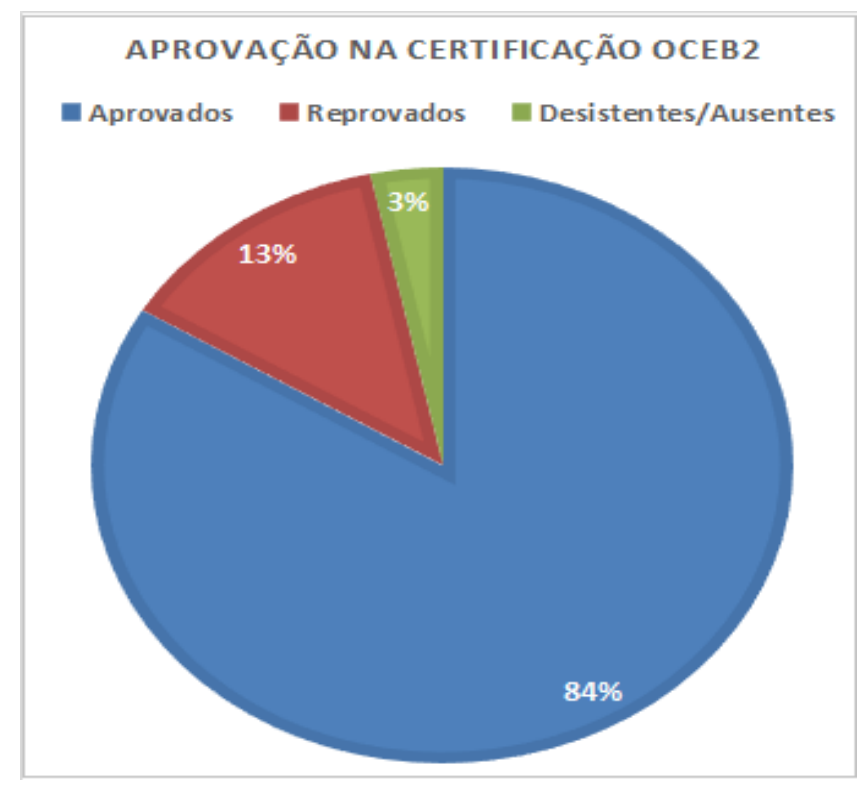

Figura 5: Taxa de aprovação dos candidatos

De modo geral, analisando todos os dados (pré e pós aplicação do exame de certificação) é possível concluir que o sistema OCEBSchool apresenta uma boa aceitação frente aos participantes, proporcionando consideráveis níveis de satisfação e comodidade em relação ao seu uso, possuindo níveis considerados aceitáveis frente a critérios de acesso, utilização, conteúdo, feedback de informações, entre outros aspectos.

\section{Conclusão e Trabalho Futuros}

No contexto da aprendizagem móvel verifica-se uma carência de aplicações simples, flexíveis, bem estruturadas e gratuitas, que proporcionem um apoio ao estudo adequado por meio de dispositivos móveis e que, ao mesmo tempo, não sejam impactadas pelas limitações desses dispositivos. Assim, este trabalho teve como objetivo o desenvolvimento de um sistema educacional móvel para o ensino e preparação da certificação OCEB, contemplando diretrizes, conceitos e exemplificações sobre a área de BPM.

Os conteúdos educacionais associados ao OCEBSchool foram modelados por meio da abordagem AIM-CID, com o auxílio de especialistas da área, havendo uma preocupação na parte de conteúdo, estrutura e exercícios/simulados. Esta modelagem, integrada a um sistema de aprendizagem, facilita o entendimento por parte dos aprendizes, principalmente em relação aos temas teóricos e abstratos, como é o caso do BPM.

Em relação aos pontos positivos, o sistema apresenta um layout bem estruturado, garantindo maior usabilidade, cognição e facilidade de uso por parte dos usuários. Os pontos limitantes identificados e analisados ao longo da avaliação de modo geral foram: (1) ausência de um tutorial auto explicativo; e (2) ausência de versões do sistema para outros sistemas operacionais móveis.

Como trabalhos futuros, pretende-se modificar e evoluir o sistema com a adição de novos módulos educacionais (seguindo a evolução da certificação OCEB 
V Congresso Brasileiro de Informática na Educação (CBIE 2016)

Anais do XXVII Simpósio Brasileiro de Informática na Educação (SBIE 2016)

foundation). Além disso, pretende-se realizar em curto prazo a incorporação de novos componentes e estrutura em relação aos outros níveis da certificação OCEB (Business/Technical Advanced e Intermediate), possibilitando assim uma sequência lógica de preparação e formação continuada aos profissionais desta área.

\section{Referências}

Barbosa, E. F. and Maldonado, J. C. (2011). Collaborative development of educationa lmodules: a need for lifelong learning. In Magoulas, G. D., editor,E-Infrastructures and Technologies for Lifelong Learning: Next Generation Environments,pages 175-211. IGI Global, Birkbeck College, University of London, UK.

Duarte, A. F.; Duarte Filho, N. F.; Barbosa, E. F.; Costa, N. P. O. (2013). Protótipo de Apoio ao Ensino e Aprendizagem de Endometriose por meio de Dispositivos Móveis. In: XXIV Simpósio Brasileiro de Informática na Educação.

Kinshuk, T., Suhonen, J., Sutinen, E., Goh, T. (2003). Mobile technologies in support of distance learning. In:Asian Journal of Distance Education, v. 1, n. 1, p. 60 -68.

Kukulska-Hulme, A., Traxler, J. (2005). Mobile teaching and learning. In: Js Mobile teaching and learning. p. 25-44.

Mates, A., (2012). BPM - Viabilidade e benefícios de gestão de processos de negocio. 1.ed. Belo Horizonte: 2012.

Moura, A. (2009). Geração Móvel: um ambiente de aprendizagem suportado por tecnologias móveis para a Geração Polegar. In P. Dias, A. J. Osório (Org.) Actas da VI Conferência Internacional de TIC na Educação Challenges 2009 / Desafios 2009. Braga: Universidade do Minho, p. 50-78.

Ozdamli, F., Cavus, N. (2011). Basic elements and characteristics of mobile learning. World Conference on Educational Technology Researches, Volume 28, p 937-94.

Rachid, L. C., Ishitani, L. (2012). m-tutorial: ferramenta de autoria para desenvolvimento de tutoriais voltados para o m-learning. In: Revista Brasileira de Informática na Educação (RBIE), v.20, n.1.

Schepman, A., Rodway, P., Beattie, C., Lambert, J. (2012). An observational study of undergraduate students adoption of (mobile) note-taking software. Computers in Human Behavior, 28(2), 308-317.

Siqueira, V., (2006). Modelagem de Processos de Negocio. Universidade de São Paulo, 2006, p.9. São Paulo.

UNESCO. Policy Guidelines for Mobile Learning. 2013. Disponível em: http://unesdoc.unesco.org/images/0021/002196/219641E.pdf>. Acesso em: 28 jan. 2014.

Zare, S. (2011). Personalization in Mobile Learning for People with Special Needs. UAHCI'11 Proceedings of the 6th international conference on Universal access in human-computer interaction: applications and services - Volume Part IV, p 662-669. 